\title{
In-Depth Relationships between Emotional Intelligence and Personality Traits in Meditation Practitioners
}

\author{
Soo-Hee Choi ${ }^{1,2}$, Seung Chan $\mathrm{An}^{3}$, UI Soon Lee ${ }^{4}$, Je-Yeon Yun ${ }^{1,5}$, Joon Hwan Jang ${ }^{1,5}$, Do-Hyung Kang ${ }^{1,2}$ \\ ${ }^{1}$ Department of Psychiatry, Seoul National University Hospital, Seoul, ${ }^{2}$ Department of Psychiatry and Institute of Human Behavioral Sciences, \\ Seoul National University College of Medicine, Seoul, ${ }^{3}$ Korea Institute of Brain Science, Seoul, ${ }^{4}$ Department of Brain Education, Global Cyber \\ University, Cheonan, ${ }^{5}$ Department of Medicine, Seoul National University College of Medicine, Seoul, Korea
}

\begin{abstract}
Objective: Meditation can elicit trait-like changes in psychological and social styles, as well as enhancement of emotional regulatory capacity. We investigated the relation between personality traits and emotional intelligence in meditation practitioners.

Methods: Seventy-two long-term practitioners of mind-body training (MBT) and 62 healthy comparative individuals participated in the study. The participants completed emotional intelligence questionnaires and the Myers-Briggs Type Indicator (MBTI).

Results: The MBT group revealed higher scores on all five emotional intelligence factors than did those in the control group, such as emotional awareness and expression, empathy, emotional thinking, emotional application, and emotional regulation (all $p \leq 0.001)$. MBT practitioners also had higher scores on the intuition of perceiving function $(t=-2.635, p=0.010)$ and on the feeling of the judging function $(t=-3.340, p=0.001)$ of the MBTI compared with those in the control group. Only the MBT group showed a robust relationship with every factor of emotional intelligence and MBTI-defined intuitive styles, indicating that higher scores of emotional intelligence were related to higher scores for intuition.

Conclusion: Emotional intelligence of meditation practitioners showed notable relationships with some features of personality trait. In-depth associations between emotional intelligence and personality traits would help to foster psychological functions in meditation practitioners.
\end{abstract}

KEY WORDS: Meditation; Mind-body training; Emotional intelligence; Personality; Intuition.

\section{INTRODUCTION}

Meditation constitutes a family of complex emotional and attentional regulatory training practices aimed at improving an individual's core psychological capacity, alleviation of stress, and/or achieving spiritual and religious goal. ${ }^{1,2)}$ Various forms of mediation, such as mindfulness meditation, mantra meditation, yoga, tai chi, and chi gong, have been used to cultivate well-being and emotional balance. ${ }^{3)}$ For example, those trained in mindfulness-based stress reduction showed decreased negative

Received: March 31, 2017 / Revised: May 2, 2017

Accepted: May 8, 2017

Address for correspondence: Do-Hyung Kang, MD, PhD Department of Psychiatry, Seoul National University Hospital, 101 Daehak-ro, Jongno-gu, Seoul 03080, Korea

Tel: +82-2-2072-0690, Fax: +82-2-744-7241

E-mail: basuare@hanmail.net

ORCID: https://orcid.org/0000-0002-8741-5748 emotional experiences during a breath-focused attention task, suggesting that this training may reduce emotional reactivity while enhancing emotional regulation. ${ }^{4)}$ Even brief meditation, for a short duration, can reduce perceived stress and improve a negative mood, ${ }^{5,6)}$ as well as change the interaction between the central and autonomic nervous systems. ${ }^{7)}$

The potential regulatory function of meditation on attentional and emotional processes can have a long-term impact on the brain and behavior in terms of changes in traits and relatively short-term states. ${ }^{8,9)}$ Improved selfmonitoring and self-regulation have been reported as long-term trait effects of engaging meditation training. ${ }^{8,10)}$ Long-term meditation practitioners also reveal different personality profiles compared with those of control subjects, such as increased extraversion and openness to experience on the Neuroticism-Extraversion-Openness FiveFactor Inventory. ${ }^{11)}$ Recent neuroscience of medita-

(ㄷ) This is an Open-Access article distributed under the terms of the Creative Commons Attribution Non-Commercial License (http://creativecommons.org/licenses/by-nc/4.0) which permits unrestricted non-commercial use, distribution, and reproduction in any medium, provided the original work is properly cited. 
tion $^{2,10)}$ imply that enduring changes in baseline or default modes of brain functioning in regions related to internalized attention may explain the trait-like transformation. ${ }^{1,12)}$

In this study, we aimed to investigate whether personality traits of meditation practitioners are associated with emotional regulatory capacity or not. The effects of mind-body training (MBT), a type of moving meditation, on stress reduction and positive affect, ${ }^{13)}$ plasma catecholamines and oxidative stress response, ${ }^{14,15)}$ and plastic changes in brain structure and function have been studied. ${ }^{12,16)}$ The present study examined emotional intelligence and the Myers-Briggs Type Indicator (MBTI) to assess emotional regulation capacity, personality traits, and their relationships in long-term meditation practitioners.

\section{METHODS}

\section{Participants}

The MBT group consisted of 72 participants who practiced meditation regularly, and the control group consisted of 62 healthy individuals who had no experience with meditation or yoga. The Structured Clinical Interview for the Diagnostic and Statistical Manual, Fourth Edition-Non-patient Edition, was used to assess psychiatric disorders in the participants. Exclusion criteria included history of a psychiatric illness, substance abuse or dependence, significant head injury, seizure disorder, or mental retardation. This study was approved by the Institutional Review Board at Seoul National University Hospital (No. 1003-061-313), and informed consent was obtained from all participants. This study was conducted in accordance with the Declaration of the World Medical Association.

Participants in the MBT group had been practicing MBT for a median of 36 months (range, 3-144 months). Most of participants were engaging in meditation for a long-term over one year. This study included participants who were homogeneous in age and who practiced the same type of meditation, called "Brain Wave Vibration" MBT. This MBT is designed to facilitate relaxation of the mind and release of negative emotions through natural rhythmic movements and a focus on bodily sensations. For this purpose, the method emphasizes heightened awareness of the movement of energy within the body by concentrating on bodily sensations and emotions. The detailed methods have been described in our previous report. ${ }^{13)}$

\section{Emotional Intelligence}

Emotional intelligence represents an individual's ability to perceive, facilitate, understand, and manage emotions in a proper and compromising manner. ${ }^{17)}$ An emotionally intelligent person is successful in adapting to circumstances that elicit emotions, either through effective regulation of the emotion itself or by applying more general coping and interpersonal skills. ${ }^{18)}$

Emotional intelligence was measured using the Korean version of the instrument ${ }^{19)}$ developed based on the ability model of emotional intelligence. ${ }^{20)}$ Emotional intelligence was measured with 50 questions categorized into five simplified factors of emotional awareness and expression (EAE), empathy (EP), emotional thinking (ET), emotional application (EA), and emotional regulation (ER). Each of these five simplified factors consisted of 10 questions describing different feelings and emotions. Each question was scored on a 5-point Likert-scale of "not at all" (1), "somewhat" (2), "moderately" (3), "very much" (4), or "absolutely" (5). Each factor was scored, and higher scores indicated greater emotional intelligence.

\section{Myers-Briggs Type Indicator}

The MBTI is a psychometric questionnaire designed to measure psychological preferences concerning perceptions of the world and decision-making. Theories about the psychological types, reflected by the MBTI and the psychological tests based on the analytic psychology of Carl Gustav Jung, ${ }^{211}$ have achieved popular consensus. Jung proposed two dichotomous pairs of cognitive functions called the "rational" (judging) functions of thinking and feeling, and the "irrational" (perceiving) functions of sensing and intuition. The MBTI reflects intrapersonal preferences, such as extraversion-introversion (E-I), sensing-intuition (S-N), thinking-feeling (T-F), and judgment-perception (J-P).

The questionnaire used in this study was the standardized MBTI Form G containing 94 items. All questionnaires were scored according to routine MBTI methods. Preferences within dichotomous pairs were transformed into one serial score. For example, an extraversion score subtracted from 100 and an introversion score of +100 were combined to create one serial score for E-I. 
Thus, low scores indicated extraversion, and high scores indicated introversion. In the same way, low scores represented sensing, thinking, and judgment, and high scores indicated intuition, feeling, and perception.

\section{Statistical Analysis}

Student's $t$ test was used to analyze differences between the two groups. Pearson's correlation coefficient analysis was used to assess the relationships between variables. In addition, partial correlation analysis was also performed to control the effect of duration of meditation, while examining the associations between emotional intelligence and personality traits. The $p$ values $<0.05$ were considered significant.

\section{RESULTS}

\section{Descriptive Characteristics}

Table 1 shows that there were no significant group differences in the demographic characteristics of age, gender, educational level, and socioeconomic status. The MBT group showed higher scores on all five factors of emotional intelligence than did those of the control group
(Table 2). MBT practitioners showed responses reflecting greater intuition in the perceiving function and greater feeling in the judging function on the MBTI than did those of the control group (Table 2).

\section{Correlations between Emotional Intelligence and MBTI in Each Group}

We examined relationships between the five factors of emotional intelligence and the four serial MBTI scores in each group. Significant positive correlations were observed between each of the five factors of emotional intelligence and the $\mathrm{S}-\mathrm{N}$ scores on the MBTI among those in the MBT group (Fig. 1B; EAE, $r=0.267, p=0.023$; EP, $r=$ 0.288, $p=0.014$; ET, $r=0.330, p=0.005 \mathrm{EA}, r=0.273, p=$ 0.021; ER, $r=0.321, p=0.006)$, indicating that higher scores of emotional intelligence were related to higher scores for intuition. However, no correlation was detected between emotional intelligence and the $\mathrm{S}-\mathrm{N}$ scores in the control group (Fig. 1A). A positive correlation was observed between the EP scores of emotional intelligence and the T-F scores in the control group $(r=0.278, p=$ 0.029).

All five factors of emotional intelligence were inversely

Table 1. Demographic characteristics of participants

\begin{tabular}{|c|c|c|c|c|}
\hline Variable & Healthy controls $(n=62)$ & MBT practitioners $(n=72)$ & $t / \chi^{2}$ & $p$ \\
\hline Age $(y r)$ & $25.5 \pm 3.6$ & $26.1 \pm 3.2$ & -1.035 & 0.303 \\
\hline Education (yr) & $14.8 \pm 1.5$ & $14.8 \pm 1.7^{*}$ & -0.088 & 0.930 \\
\hline Gender, male & $38(61.3)$ & $35(48.6)$ & 2.160 & 0.142 \\
\hline Socioeconomic status, I/II/III/IV/V & $1 / 23 / 22 / 8 / 2^{\dagger}$ & $0 / 13 / 27 / 2 / 1^{\ddagger}$ & 6.629 & 0.157 \\
\hline Duration of meditation (mo) & - & $42.8 \pm 3.5$ & - & - \\
\hline
\end{tabular}

Values are presented as mean \pm standard deviation, number (\%), or number only.

Class I of socioeconomic status represents the highest level and Class $\vee$ represents the lowest level.

${ }^{*} \mathrm{n}=71,{ }^{\dagger} \mathrm{n}=56,{ }^{\dagger} \mathrm{n}=43$.

Table 2. Group differences in emotional intelligence and four functions on the Myers-Briggs Type Indicator

\begin{tabular}{|c|c|c|c|c|}
\hline Variable & Healthy controls $(n=62)$ & MBT practitioners $(n=72)$ & $t$ & $p$ \\
\hline \multicolumn{5}{|l|}{ Emotional intelligence } \\
\hline Emotional awareness and expression & $34.7 \pm 5.4$ & $37.8 \pm 4.0$ & -3.756 & $<0.001$ \\
\hline Empathy & $34.2 \pm 5.0$ & $36.9 \pm 3.6$ & -3.458 & 0.001 \\
\hline Emotional thinking & $32.9 \pm 5.3$ & $39.5 \pm 4.1$ & -8.138 & $<0.001$ \\
\hline Emotional application & $33.0 \pm 4.7$ & $36.3 \pm 5.2$ & -3.887 & $<0.001$ \\
\hline Emotional regulation & $33.4 \pm 5.9$ & $37.5 \pm 4.9$ & -4.408 & $<0.001$ \\
\hline \multicolumn{5}{|l|}{ Myers-Briggs Type Indicator } \\
\hline Extraversion-Introversion & $94.2 \pm 24.3$ & $97.1 \pm 24.0$ & -0.692 & 0.490 \\
\hline Sensing-Intuition & $86.1 \pm 23.1$ & $95.4 \pm 17.0$ & -2.635 & 0.010 \\
\hline Thinking-Feeling & $87.0 \pm 20.7$ & $98.6 \pm 19.4$ & -3.340 & 0.001 \\
\hline Judgment-Perception & $95.1 \pm 31.1$ & $99.9 \pm 25.8$ & -0.986 & 0.326 \\
\hline
\end{tabular}

Values are presented as mean \pm standard deviation. 
A

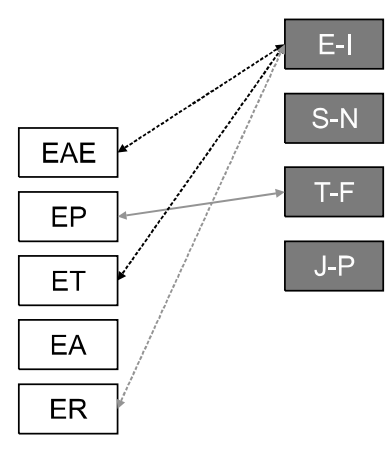

Control $(n=62)$
B

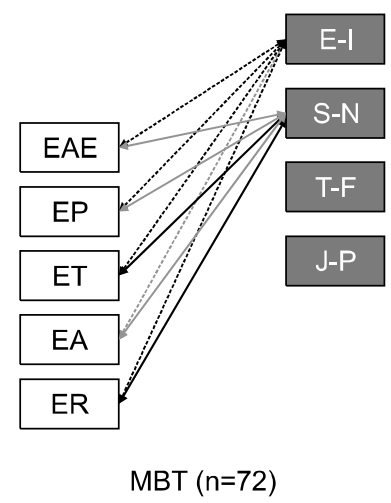

Fig. 1. Correlations between emotional intelligence and MyersBriggs Type Indicator (MBTI) scores in the control (A) and MBT (B) groups. E, S, T, and J indicate low serial scores and I, N, F, and P indicate high serial scores.

Dotted arrows, negative correlations; line arrows, positive correlations; gray-colored arrows, $p<0.05$; black-colored arrows, $p<0.01$. $\mathrm{MBT}$, mind-body training; $\mathrm{EAE}$, emotional awareness and expression; $E P$, empathy; ET, emotional thinking; EA, emotional application; ER, emotional regulation; $\mathrm{E}-\mathrm{I}$, extraversion-introversion; $\mathrm{S}-\mathrm{N}$, sensingintuition; T-F, thinking-feeling; J-P, judgment-perception.

correlated with the E-I scores in the MBT group (Fig. 1B; $\mathrm{EAE}, r=-0.487, p<0.001 ; \mathrm{EP}, r=-0.384, p=0.001 ; \mathrm{ET}$, $r=-0.444, p<0.001 ; \mathrm{EA}, r=-0.297, p=0.011 ; \mathrm{ER}, r=$ $-0.306, p=0.009$ ), indicating that the higher scores for all components of emotional intelligence were associated with a high level of extraversion. In the control group, three factors of emotional intelligence were inversely correlated with E-I scores (Fig. 1A; EAE, $r=-0.401, p=0.001$; $\mathrm{ET}, r=-0.394, p=0.002$; $\mathrm{ER}, r=-0.292, p=0.021$ ).

Above associations were remained significant, when duration of the MBT was controlled in partial correlation analyses.

\section{DISCUSSION}

The MBT practitioners demonstrated consistent relationships between every component of emotional intelligence and extraverted and intuitive types on the MBTI. In addition, they nor only showed the higher levels of intuition in forming perceptions and using feelings for making judgments in personality traits, but also enhanced emotional intelligence compared to the control group.

Compassion meditation experts who have cultivate empathy and theory of mind for less than 10 years showed altered activation of related brain circuitry, suggesting that

a repeated practice could result in enduring change with regard to affective and social style. ${ }^{9)}$ Considering that the 8-week of MBT showed a positive effect on emotional intelligence, $\left.{ }^{6}\right)$ it is likely that this study population of longterm meditators practicing over one year would also have an enduring trait-like change as other kinds of meditations. MBT, which combines ancient Eastern philosophy with modern scientific methods, is designed to relax the mind and body and to elevate awareness. ${ }^{12)}$ MBT helps to quiet the thinking mind and release negative emotions by performing natural rhythmic movements. ${ }^{13)}$ As part of this process, MBT places importance on focusing attention on emotions and bodily sensations. ${ }^{12)}$ The somatic marker hypothesis describes a mechanism by which our behavior can be guided by emotion and shows that this emotion arises from body feedback. ${ }^{22)}$ MBT practitioners, who have learned the interaction between mind and the body, acquire reasonable proficiency in using their body signals to perceive and facilitate their emotions, which can be reflected on the higher emotional intelligence of the MBT group.

The MBTI-defined intuitive type is associated with a preference to focus on the future and perceive possibilities, whereas the sensing type is associated with a preference to focus on the present and use concrete information. ${ }^{23)}$ Dual-process theories that distinguish a quick, intuitive, and effortless processing style from a slow, analytical, and deliberate reasoning style ${ }^{24)}$ have been associated with functional activity in different brain regions. ${ }^{25)}$ In particular, effortless intuition is related to activities in the insula and anterior cingulate cortex. ${ }^{25)}$ Furthermore, the caudate is activated during quick automatic processes of intuition in experts, and activation of the caudate develops in novices after intuition training. ${ }^{26,27)}$ These subcortical areas related to intuition are closely associated with the limbic system, which supports a variety of functions including emotions. Thus, the distinct relationship between intuitive aptitude and emotional intelligence, which was observed in the MBT group, suggests that meditation practitioners can utilize their emotional intelligence for intuitive perception. As meditation can alter interactions between the central and autonomic nervous systems, ${ }^{7}$ MBT seems to improve the efficiency of interactions between the emotional and intuitive aspects involved in perceiving irrational functions.

In addition, previous studies have reported increased 
dopamine release from the striatum during Yoga Nidra meditation $^{28)}$ and an increase in the plasma dopamine level among MBT practitioners. ${ }^{13)}$ Considering a previous report that "gut-level" intuition may be related to the basal ganglia-dopamine system, ${ }^{29)}$ this suggests that a greater reliance on intuition as the basis for perceiving in the MBT group may also be associated with long-term meditation practice. The greater preference for feelings in the MBT than that in the control group may also be associated with meditation training and increased emotional intelligence. The MBTI defines feeling types based on decisions regarding values and personal concerns, whereas thinking types base decisions on logic. ${ }^{23)}$ Feeling individuals are more subjective, and intimate; they like to use persuasion, and are personable, humane, harmony oriented, sympathetic, and devoted. ${ }^{30)}$ Given that MBTI feeling types report higher psychological well-being than that of thinking types, ${ }^{23)}$ the regulatory effects of meditation on emotional processes, which also involve decision making, affect reliance on feelings to reach a decision.

The two groups showed a similar association between emotional intelligence and the propensity for extroversion, which was considerable in both groups. Extraverts may be better able to employ emotional information, as compared to introverts, as they are stimulated rather than overwhelmed by emotion information. ${ }^{17)}$ However, the more robust relationships between the MBTI-defined extroverted types and the emotional intelligence of the MBT group, compared to those in the control group, suggest that meditation strengthens the availability of emotional information to practitioners. This observation agrees with previous findings that extraversion is positively correlated with practicing meditation and that increasing emotional competence leads to long-term significant increases in extraversion. ${ }^{31,32)}$

The present findings are limited by the cross-sectional design of this study, precluding an inference of the cause-effect relationships. Whether the associations between emotional intelligence and personality traits are the baseline characteristics of meditators or the influence of medication can be found out by a follow-up research while controlling for the pre-existing personality traits, lifestyle and other regular activities. Thus, the additional information on the baseline characteristics of the participants should be provided. Second, in this study population of long-term meditators, it is difficult to explore a dose-response relationship in trait-like changes with duration of meditation. Further research on a heterogeneous group of meditators would be needed to find linear relationships between duration and effect of meditation. Furthermore, although there was no statistical group-difference in the gender composition, a considerable difference of the two groups should be considered in the interpretation of the results. Finally, emotional intelligence and personality were assessed by self-reported measures. Emotional intelligence is related to personality traits, and self-report measures overlap more with personality measures. ${ }^{33)}$ However, the contributions of emotional intelligence to academic, social, and career outcomes go beyond the contributions of personality, suggesting that emotional intelligence is distinct from the major dimensions of personality. ${ }^{17)}$

In conclusion, MBT practitioners showed increased emotional intelligence, which was strongly correlated with extroverted and intuitive types of MBTI personality traits. The specific goal of the MBT, which cultivates emotional and bodily awareness and their interaction, is closely related to emotional intelligence. This emotional regulatory capacity, which involves ability to carry out accurate reasoning about emotions and use emotions to enhance thoughts and behavior, ${ }^{17)}$ can play a key role in non-pharmacological interventions in mental disorders such as cognitive-behavioral therapy. When increased emotional intelligence is connected to trait-like changes or enduring patterns of inner experience and behavior, therapeutic effects might be improved and/or maintained on mental disorders. A follow-up study on the impact of meditation in patients with depression, suicidality, or distress with general medication conditions ${ }^{30,34,35)}$ would help in developing alternative solving approaches in psychopathology.

\section{- Acknowledgments}

All authors report no financial relationship with any commercial interest. This research was supported by the Brain Research Program through the National Research Foundation of Korea (NRF) funded by the Ministry of Science, ICT \& Future Planning (NRF-2016M3C7A1914449, http://www.nrf.re.kr). The funders had no role in study design, data collection and analysis, decision to publish, or preparation of the manuscript. 


\section{REFERENCES}

1. Lutz A, Slagter HA, Dunne JD, Davidson RJ. Attention regulation and monitoring in meditation. Trends Cogn Sci 2008; 12:163-169.

2. Tang YY, Hölzel BK, Posner MI. The neuroscience of mindfulness meditation. Nat Rev Neurosci 2015;16:213-225.

3. Ospina MB, Bond K, Karkhaneh M, Tjosvold L, Vandermeer B, Liang $\mathrm{Y}$, et al. Meditation practices for health: state of the research. Evid Rep Technol Assess (Full Rep) 2007;(155): 1-263.

4. Goldin PR, Gross JJ. Effects of mindfulness-based stress reduction (MBSR) on emotion regulation in social anxiety disorder. Emotion 2010;10:83-91.

5. Lane JD, Seskevich JE, Pieper CF. Brief meditation training can improve perceived stress and negative mood. Altern Ther Health Med 2007;13:38-44.

6. Jung $\mathrm{YH}, \mathrm{Ha} \mathrm{TM}, \mathrm{Oh} \mathrm{CY}$, Lee US, Jang JH, Kim J, et al. The effects of an online mind-body training program on stress, coping strategies, emotional intelligence, resilience and psychological state. PLoS One 2016;11:e0159841.

7. Tang YY, Ma Y, Fan Y, Feng H, Wang J, Feng S, et al. Central and autonomic nervous system interaction is altered by short-term meditation. Proc Natl Acad Sci U S A 2009;106: 8865-8870.

8. Cahn BR, Polich J. Meditation states and traits: EEG, ERP, and neuroimaging studies. Psychol Bull 2006;132:180-211.

9. Lutz A, Brefczynski-Lewis J, Johnstone T, Davidson RJ. Regulation of the neural circuitry of emotion by compassion meditation: effects of meditative expertise. PLoS One 2008;3: e1897.

10. Lutz A, Greischar LL, Rawlings NB, Ricard M, Davidson RJ. Long-term meditators self-induce high-amplitude gamma synchrony during mental practice. Proc Natl Acad Sci U S A 2004; 101:16369-16373.

11. Jung $\mathrm{YH}$, Lee US, Jang JH, Kang DH. Effects of mind-body training on personality and behavioral activation and inhibition system according to BDNF Val66Met polymorphism. Psychiatry Investig 2016;13:333-340.

12. Jang JH, Jung $\mathrm{WH}$, Kang $\mathrm{DH}$, Byun MS, Kwon $\mathrm{SJ}$, Choi $\mathrm{CH}$, et al. Increased default mode network connectivity associated with meditation. Neurosci Lett 2011;487:358-362.

13. Jung $\mathrm{YH}$, Kang DH, Jang JH, Park HY, Byun MS, Kwon SJ, et al. The effects of mind-body training on stress reduction, positive affect, and plasma catecholamines. Neurosci Lett 2010;479: 138-142.

14. Jung $\mathrm{YH}$, Kang DH, Byun MS, Shim G, Kwon SJ, Jang GE, et al. Influence of brain-derived neurotrophic factor and catechol O-methyl transferase polymorphisms on effects of meditation on plasma catecholamines and stress. Stress 2012;15:97-104.

15. Lee DH, Park HY, Lee US, Lee KJ, Noh EC, Jang JH, et al. The effects of brain wave vibration on oxidative stress response and psychological symptoms. Compr Psychiatry 2015;60: 99-104.
16. Kang $\mathrm{DH}$, Jo $\mathrm{HJ}$, Jung $\mathrm{WH}$, Kim $\mathrm{SH}$, Jung $\mathrm{YH}$, $\mathrm{Choi} \mathrm{CH}$, et al. The effect of meditation on brain structure: cortical thickness mapping and diffusion tensor imaging. Soc Cogn Affect Neurosci 2013;8:27-33.

17. Mayer JD, Roberts RD, Barsade SG. Human abilities: emotional intelligence. Annu Rev Psychol 2008;59:507-536.

18. Bhullar N, Schutte NS, Malouff JM. The nature of well-being: the roles of hedonic and eudaimonic processes and trait emotional intelligence. J Psychol 2013;147:1-16.

19. Moon Y. Report of the Saehan Media EQ test. Seoul:Saehan Media;1999.

20. Mayer JD, Salovey P. What is emotional intelligence? In: Salovey $P$, Sluyter $D$, editors. Emotional development and emotional intelligence: educational implications. New York: Basic Books; 1997. p.3-34.

21. Jung CG. Psychological types: volume 6 of the collected works of CG Jung. Princeton, NJ:Princeton University Press;1971. p. 169-170.

22. Dunn BD, Dalgleish T, Lawrence AD. The somatic marker hypothesis: a critical evaluation. Neurosci Biobehav Rev 2006; 30:239-271.

23. Harrington R, Loffredo DA. The relationship between life satisfaction, self-consciousness, and the Myers-Briggs type inventory dimensions. J Psychol 2001;135:439-450.

24. Alter AL, Oppenheimer DM, Epley N, Eyre RN. Overcoming intuition: metacognitive difficulty activates analytic reasoning. J Exp Psychol Gen 2007; 136:569-576.

25. Kuo WJ, Sjöström T, Chen YP, Wang YH, Huang CY. Intuition and deliberation: two systems for strategizing in the brain. Science 2009;324:519-522.

26. Wan X, Takano D, Asamizuya T, Suzuki C, Ueno K, Cheng K, et al. Developing intuition: neural correlates of cognitive-skill learning in caudate nucleus. J Neurosci 2012;32:1749217501.

27. Wan X, Nakatani H, Ueno K, Asamizuya T, Cheng K, Tanaka $\mathrm{K}$. The neural basis of intuitive best next-move generation in board game experts. Science 2011;331:341-346.

28. Kjaer TW, Bertelsen C, Piccini P, Brooks D, Alving J, Lou HC. Increased dopamine tone during meditation-induced change of consciousness. Brain Res Cogn Brain Res 2002;13:255259.

29. Frank MJ, O'Reilly RC, Curran T. When memory fails, intuition reigns: midazolam enhances implicit inference in humans. Psychol Sci 2006; 17:700-707.

30. Janowsky DS, Hong E, Morter S, Howe L. Myers Briggs Type indicator personality profiles in unipolar depressed patients. World J Biol Psychiatry 2002;3:207-215.

31. Nelis D, Kotsou I, Quoidbach J, Hansenne M, Weytens F, Dupuis $\mathrm{P}$, et al. Increasing emotional competence improves psychological and physical well-being, social relationships, and employability. Emotion 2011;11:354-366.

32. Delmonte MM. Personality correlates of meditation practice frequency and dropout in an outpatient population. J Behav 
Med 1988; 11:593-597.

33. Di Fabio A, Kenny ME. Promoting well-being: the contribution of emotional intelligence. Front Psychol 2016;7: 1182.

34. Janowsky DS, Morter S, Hong L. Relationship of Myers Briggs type indicator personality characteristics to suicidality in affective disorder patients. J Psychiatr Res 2002;36:33-39.

35. Ludwig DS, Kabat-Zinn J. Mindfulness in medicine. JAMA 2008;300:1350-1352. 